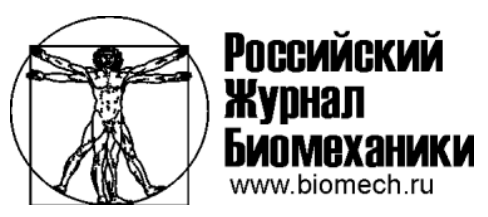

\title{
ИНТРАМЕДУЛЛЯРНЫЙ СТЕРЖЕНЬ НОВОГО ТИПА ДЛЯ ОСТЕОСИНТЕЗА ДИАФИЗАРНЫХ ПЕРЕЛОМОВ БЕДРА
}

\author{
Д.В. Иванов ${ }^{1}$, А.П. Барабаш ${ }^{2}$, Ю.А. Барабаш ${ }^{2}$ \\ ${ }^{1}$ Отдел математического моделирования образовательно-научного института наноструктур и биосистем \\ Саратовского государственного университета имени Н.Г. Чернышевского, Россия, 410012, Саратов, \\ ул. Астраханская, 83, e-mail: ivanovdv@gmail.com \\ 2 Отдел инновационных проектов в травматологии и ортопедии Саратовского научно-исследовательского \\ института травматологии и ортопедии Минздрава России, Россия, 410002, Саратов, ул. Чернышевского, \\ 148, e-mail: yubarabash@yandex.ru
}

\begin{abstract}
Аннотация. В настоящее время интрамедуллярный остеосинтез блокируемыми стержнями является наиболее распространенным методом лечения диафизарных переломов длинных костей нижней конечности человека. Современные расширяющиеся стержни, такие как Fixion, имеют ряд преимуществ перед стандартными блокируемыми. Они быстро устанавливаются и обеспечивают необходимую аутокомпрессию на стыке отломков. Тем не менее исследования показывают их невысокую стабильность при крутильных нагрузках. Более того, такие стержни существенно дороже стандартных. В данной работе предлагается новая конструкция интрамедуллярного стержня. Проведено конечно-элементное моделирование напряженно-деформированного состояния системы «бедренная кость-фриксатор» при трех внешних нагрузках (осевой, поперечной и скручивающей), прикладываемых к головке кости. Исследованы два фриксатора (разработанный и стандартный фирмы ChM) и два типа диафизарных переломов A1 (косой винтообразный) и B2 (оскольчатый с одним центральным осколком) в соответствии с классификацией $A O / A S I F$. Выявлено, что разрабатываемый интрамедуллярный фиксатор обеспечивает осевую жесткость от 428 до $510 \mathrm{H} / \mathrm{Mm}$ для переломов $A 1$ и B2. Результаты расчетов показали равномерное распределение контактного давления между костными отломками. Дистальный блокирующий винт нового стержня при всех исследованных нагрузках не испытывал критических напряжений, основная нагрузка распределялась на тело стержня и его лопасти. Разрабатываемый стержень не имеет существенных недостатков блокируемого и расширяющегося стержней. Новый фиксатор обеспечивает высокую стабильность при скручивании и равномерную аутокомпрессию на стыке отломков, а также более плотный контакт отломков.
\end{abstract}

Ключевые слова: интрамедуллярный остеосинтез, конечно-элементное моделирование, перелом бедра, эффективные напряжения, контактное давление.

\section{ВведенИЕ}

На сегодняшний день остеосинтез блокируемыми интрамедуллярными стержнями является «золотым стандартом» лечения диафизарных переломов длинных костей нижней конечности человека [21]. Эта методика позволяет быстро

(С) Иванов Д.В., Барабаш А.П., Барабаш Ю.А., 2015

Иванов Дмитрий Валерьевич, к.ф.-м.н., заместитель начальника отдела математического моделирования, Саратов

Барабаш Анатолий Петрович, д.мед.н., профессор, руководитель отдела новых технологий в травматологии, Саратов

Барабаш Юрий Анатольевич, д.мед.н., в.н.с. отдела новых технологий в травматологии, Саратов 
стабилизировать перелом с помощью относительно малоинвазивной процедуры по установке и вернуть поврежденную конечность к полноценному функционированию $[1,6,7,10]$. Расширяющиеся стержни, такие как Fixion, являются относительно новой технологической разработкой и устанавливаются без помощи проводника и рассверливания канала. По мнению авторов $[12,14]$, они обеспечивают необходимую стабильность перелома, что позволяет не закреплять стержень с помощью блокирующих винтов.

Ретроспективное сравнительное исследование показало преимущества расширяющегося стержня перед блокируемым при остеосинтезе диафизарных переломов бедренных костей [13]. Расширяющийся стержень существенно ускоряет проведение операции, соответственно, уменьшается вредное воздействие радиационного излучения на человека, что особенно важно для пациентов с множественными травмами и переломами. При этом авторы отмечают существенно более высокую стоимость расширяющихся стержней относительно стандартных блокируемых.

Несмотря на то что исследования расширяющихся стержней в настоящее время являются очень перспективными, во многих работах по этой тематике показаны методологические недоработкитребующие дополнительного осмысления [21]. Более того, расширяющиеся стержни могут не обеспечивать необходимую жесткость системы кость-имплантат $[15,18]$. При этом отмечаются такие послеоперационные осложнения, как разрушение стержня и несращение стержня, укорочение конечности $[18,19,22]$. Сравнительный анализ стабильности фиксации переломов с помощью стандартных блокируемых стержней и расширяющихся Fixion показал, что «- »последние хуже сопротивляются скручивающим нагрузкам [17]. Работы других авторов [3] также выявили меньшую жесткость сравнению с жесткостью на кручение.

В последние годы компьютерное моделирование все чаще используется для биомеханического исследования интрамедуллярного остеосинтеза переломов длинных трубчатых костей $[5,9,16,20,26]$. При рассмотрении блокируемых стержней многие авторы отмечают наличие концентраций напряжений на дистальных блокирующих винтах, а также на отверстиях под них в стержне [8, 25]. Например, в монографии [25] приводятся результаты численных экспериментов по моделированию остеосинтеза поперечного перелома диафиза бедренной кости интрамедуллярным блокируемым стержнем при помощи метода конечных элементов. Расчеты показали наличие высоких эффективных напряжений на блокирующих винтах. Значения достигали 400 МПа при осевой нагрузке на систему кость-фиксатор в $580 \mathrm{H}$.

Таким образом, и блокируемые, и расширяющиеся стержни имеют как преимущества, так и недостатки, не позволяющие тому или другому типу стать оптимальным интрамедуллярным фиксатором. Именно поэтому вопрос о разработке нового типа фиксатора актуален и сегодня. Эту задачу пытаются решить многие исследователи. Например, в работе [4] методом конечных элементов исследуется новый тип стержня, не имеющий блокирующих винтов. Фиксация стержня внутри костного канала должна обеспечиваться конструкцией имплантата, внутри которого находятся специальные зубчатые колеса. Ingrassia и соавт. [11] разработали новый интрамедуллярный стержень, имеющий дистальный расширяющийся конец. Такая конструкция позволила избежать блокирования стержня с помощью дистальных винтов, при этом обеспечила высокую стабильность при исследованных в работе нагрузках. В монографии [23] предлагается новый стержень, выполненный из нитинола. Авторы предполагают, что фиксатор будет расширяться за счет воздействия температуры тела человека. Стержень может быть использован для фиксации диафизарных переломов длинных трубчатых костей. 


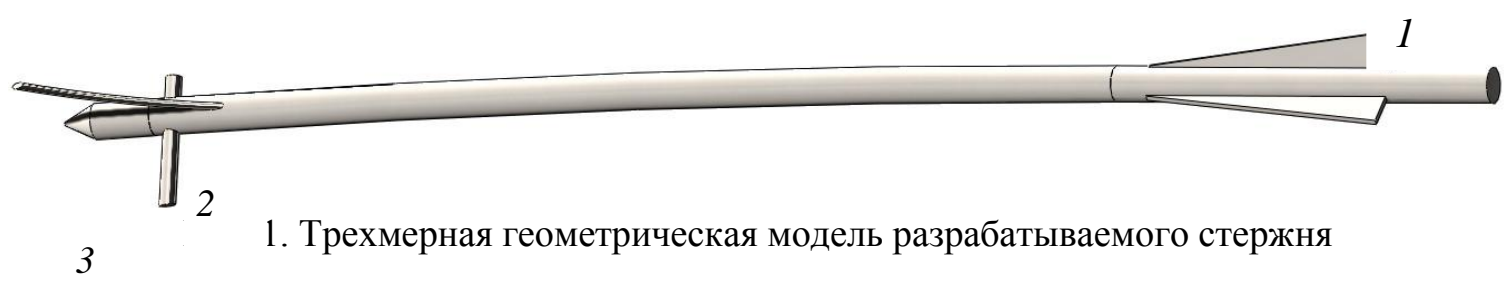

При разработке интрамедуллярных стержней исследователи стараются частично или полностью уйти от блокирующих винтов, но при этом важно понимать, что именно винты обеспечивают стабильность перелома при воздействии скручивающих нагрузок. Поэтому, по нашему мнению, полностью уходить от блокирующих винтов нельзя необходимо совместить блокирование винтами и блокирование при помощи формы стержня.

В данной работе предлагается принципиально новая конструкция интрамедуллярного стержня. Его проксимальный отдел имеет три специальных ребра и по форме напоминает «ракету». Ребра. обеспечивают жесткость на кручение, при этом проксимальный костный отломок не заблокирован в осевом направлении. Такая конструкция позволит создать на стыке отломков необходимое контактное давление для лучшего роста костной ткани и заживления перелома. .В дистальной части стержня предлагается сохранить один блокирующий винт.1 и добавить упругий элемент спицу., призванную удерживать стержень за счет фиксации в одном из мыщелков кости.

\section{МАТЕРИАЛЫ И МЕТОДЫ}

Разрабатываемый интрамедуллярный стержень (патент $\quad$ РФ № 115646 (А.П. Барабаш, И.А. Норкин, Ю.А. Барабаш) изображен на рис. 1. Его форма предполагает наличие в проксимальном отделе трех разновеликих ребер, размер и форма которых оптимизированы под анатомическое строение проксимального отдела бедренной кости. Такая конструкция практически исключает ротационные перемещения проксимального отломка. Площадь контакта стержня с костной массой при этом оказывается значительно большей, чем у стандартного блокируемого стержня

Дистальный отдел стержня имеет два отверстия. Одно из них располагается на конце и предназначено для установки кортикального винта в поперечном направлении. Второе отверстие имеет овальную форму, выполнено под углом к оси стержня и имеет связь с его центральным каналом. Через это отверстие выходит упругая спица, фиксирующаяся внутри мыщелка бедра. Материал стержня - нержавеющая сталь.

Трехмерная геометрическая модель бедренной кости с диафизарными переломами $A 1$ и $B 2$ [2] была построена в специализированном программном комплексе SolidWorks на основе поперечных срезов, полученных с помощью компьютерной томограммы. Затем стержень устанавливался в кость, и создавалась система кость-фиксатор. При моделировании предполагалось, что бедренная кость представляется трабекулярным и кортикальным слоями.

На рис. 2 изображены геометрические модели систем кость-фиксатор для диафизарных переломов $A 1$ и $B 2$.

Решались статические задачи теории упругости, описывающие напряженнодеформированное состояние систем кость-фиксатор при трех типах нагрузок: осевая $(600 \mathrm{H})$, поперечная $(100 \mathrm{H})$ и скручивающая $(10 \mathrm{H} \cdot \mathrm{m})$. Нагрузки прикладывались к головке кости. Дистальный конец кости жестко закреплялся(.)., рассмотренные в данной работе, 


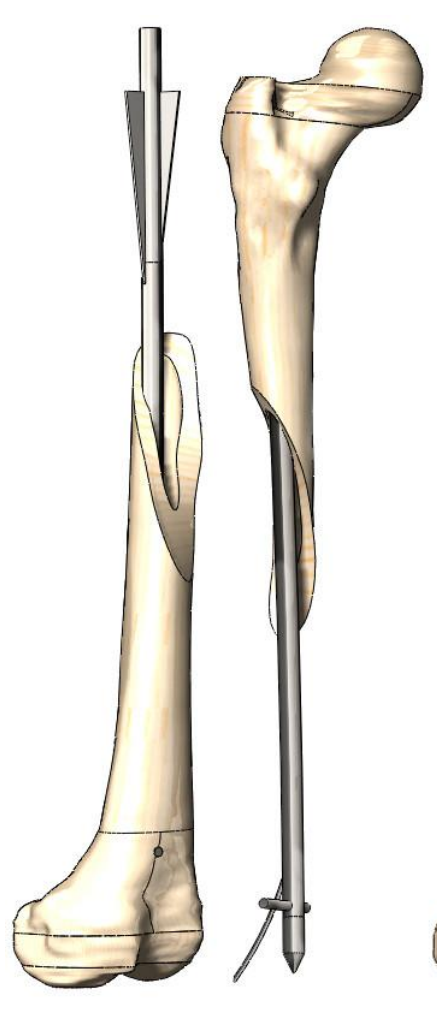

$a$

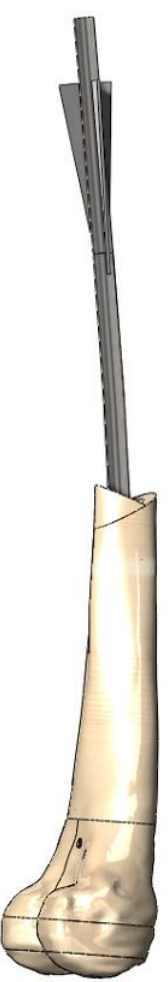

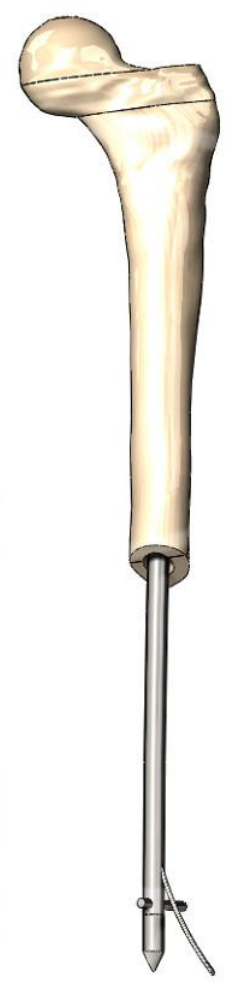

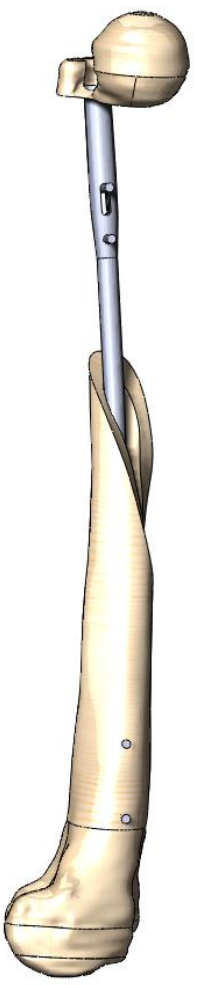

$\sigma$

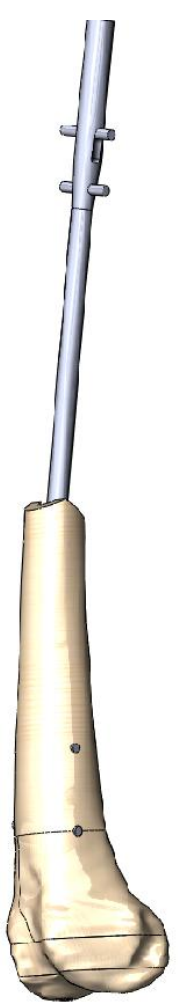

Рис. 2. Исследованные модели систем «бедренная кость - фиксатор» при переломах $A 1$ и $B 2: a$-разработанный имплантат; $\sigma$ - стандартный стержень $C h M$
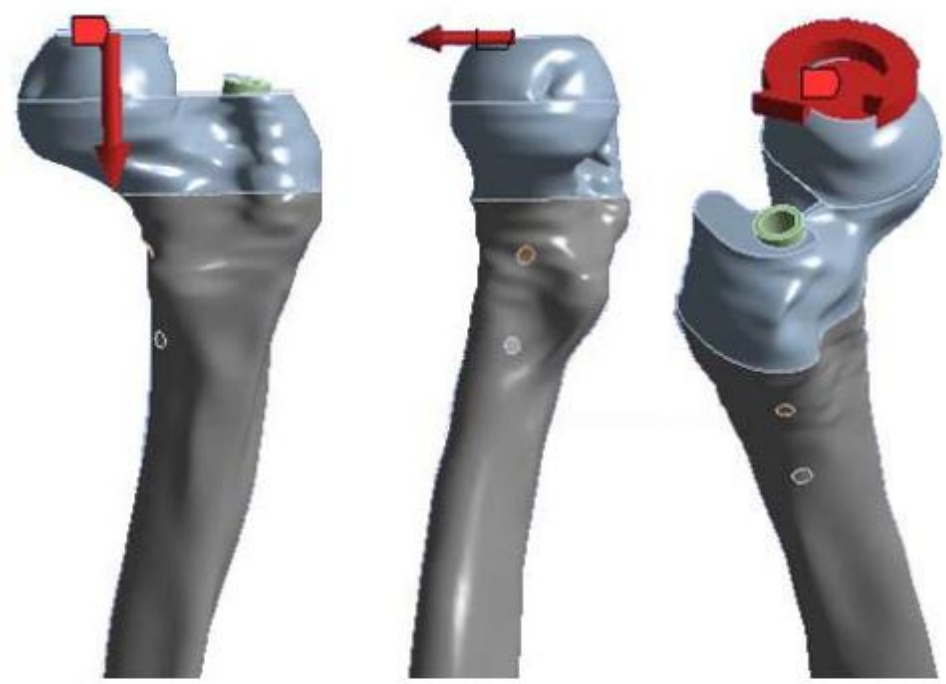

Рис. 3. Типы и места приложения исследованных нагрузок

Предполагалось, что стержень изготовлен из идеально упругого изотропного и однородного материала. Модуль упругости кортикальной кости, на $30 \%$ был больше модуля упругости трабекулярной кости [24, 27]. ,

При моделировании учитывалось контактное взаимодействие между костными отломками, осколками и имплантатом. Предполагалось, что в процессе нагружения могут возникать конечные деформации исследуемых объектов. 
Задачи решались численно в системе конечно-элементного анализа Ansys, среде Workbench. Было проведено исследование сеточной сходимости задачи для определения размера элемента вычислительной сетки, не оказывающей влияния на результаты расчетов. Было установлено, что начиная с ребра элемента длиной 0,5 мм результаты расчетов перестают быть зависимыми от качества сетки. Ввиду сложности геометрических моделей исследуемых объектов использовалась тетраэдрическая вычислительная сетка, состоящая из линейных конечных элементов.

\section{РЕЗУЛЬТАТЫ И ОБСУЖДЕНИЕ}

Предметом исследования явилось напряженно-деформированное состояние систем кость-имплантат для двух типов переломов ( $A 1$ и $B 2)$. Сравнивались и анализировались эффективные напряжения, деформации, возникающие в костных отломках, осколках и элементах имплантата, а также перемещения головки кости.

Результаты расчетов показали, что для трех исследованных нагрузок наименьшие перемещения головки кости возникают в случае скручивающей нагрузки и составляют порядка 0,6 мм. Наибольшие перемещения головки кости возникают в случае приложения поперечной силы. Их величина чуть менее 3 мм. При осевой нагрузке перемещения головки кости не превышают 1,4 мм. Данные по перемещениям головки кости для трех исследованных нагрузок, двух переломов и двух стержней приведены в таблице.

Из таблицы видно, что новый стержень показывает более высокую стабильность фиксации (жесткость системы кость-имплантат) в случае трех исследованных нагрузок и перелома $B 2$ по сравнению со стандартным стержнем (сравниваются третий и пятый столбцы таблицы, где показаны наибольшие полные перемещения головки бедра для перелома $B 2$ и обоих стержней). Что касается перелома $A 1$, то здесь оба стержня показали очень схожую жесткость системы кость-имплантат, что и подтверждается схожими значениями величин полных перемещений головки бедра для трех исследованных нагрузок (см. таблицу).

Перейдем к рассмотрению распределения и величины контактного давления на стыке костных отломков. Необходимо отметить, что в случае установки стержня ChM распределение контактного давления между отломками является существенно неравномерным. Данный факт говорит о неравномерности аутокомпрессии на стыке отломков, что может быть причиной их неудачного сращения (рис. 4 и 5).

Максимальные перемещения, мм, системы кость-имплантат при моделировании осевой, поперечной и скручивающей нагрузок для переломов $A 1$ и $B 2$

\begin{tabular}{|l|c|c|c|c|}
\hline \multirow{2}{*}{ Нагрузка } & $\begin{array}{c}\text { Стержень нового поколения - } \\
\text { трехлопастной, с дистальным } \\
\text { блокирующим винтом } \\
\text { и упругой спицей }\end{array}$ & \multicolumn{2}{|c|}{$\begin{array}{c}\text { Стандартный блокируемый } \\
\text { стержень } C h M\end{array}$} \\
\cline { 2 - 5 } & Перелом $A 1$ & Перелом $B 2$ & Перелом $A 1$ & Перелом $B 2$ \\
\hline Осевая & 1,40 & 1,18 & 1,40 & 1,44 \\
\hline Поперечная & 2,72 & 2,95 & 2,71 & 3,36 \\
\hline Скручивающая & 0,63 & 0,62 & 0,63 & 1,20 \\
\hline
\end{tabular}




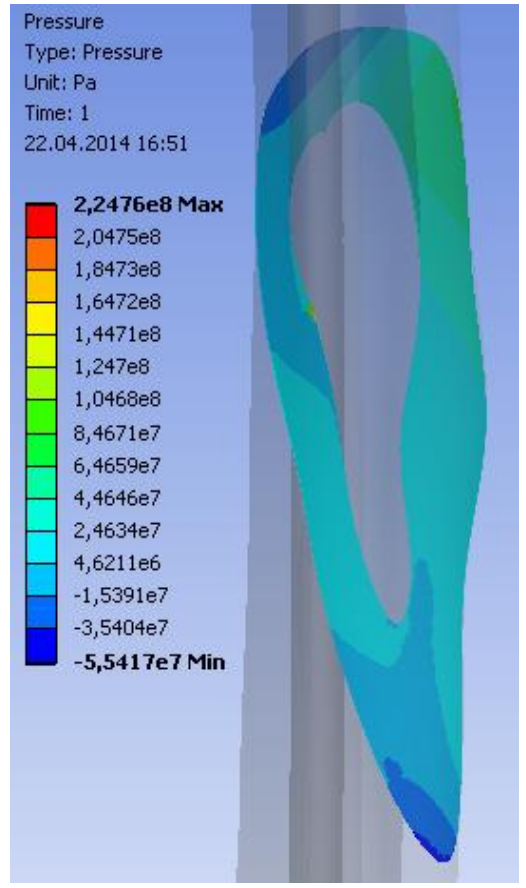

$a$

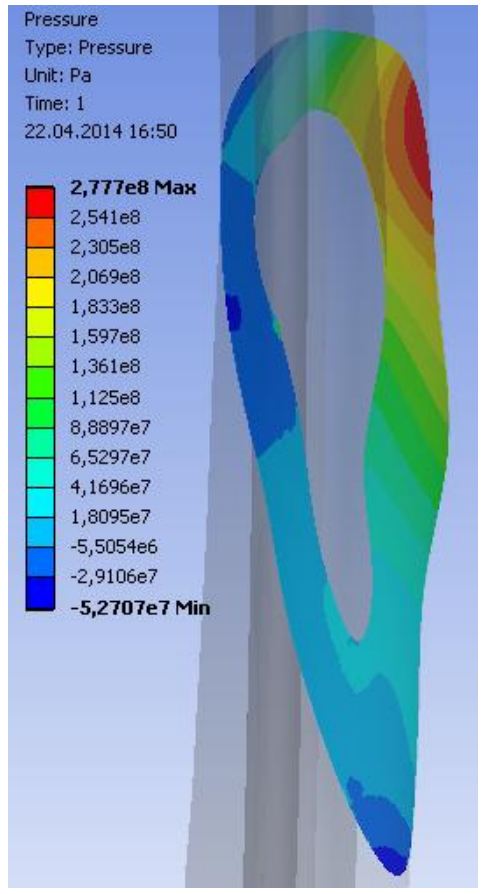

$\sigma$

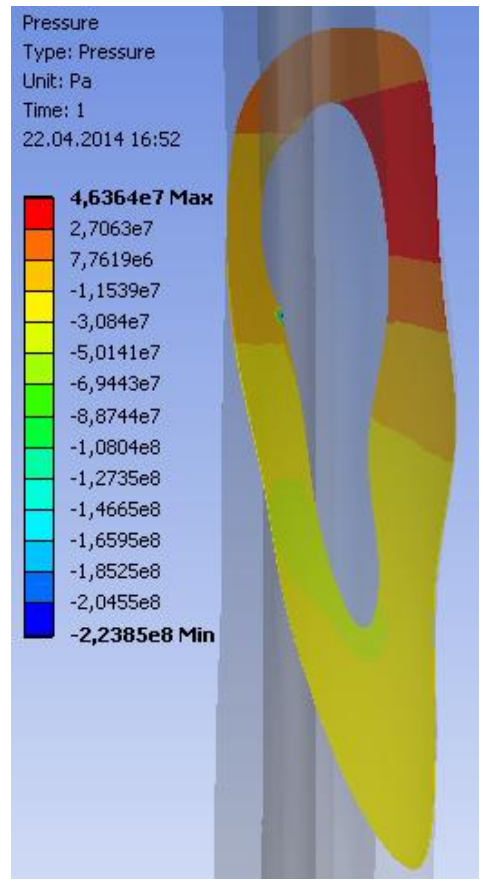

B

Рис. 4. Контактное давление между отломками для перелома $A 1$ (стандартный стержень $(h M): a$ - осевая сила; $\sigma$ - поперечная сила; 6 - скручивающий момент

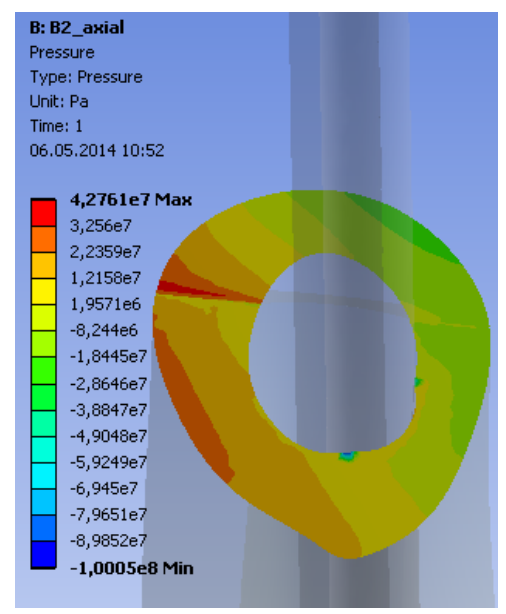

$a$
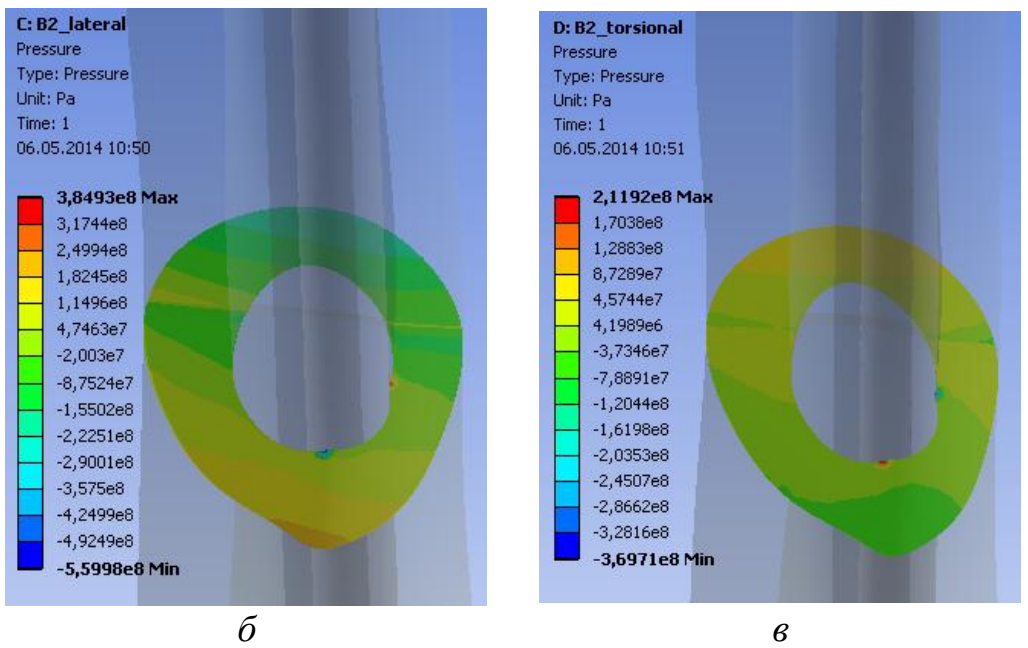

Рис. 5. Контактное давление между отломками для перелома $B 2$ (стандартный стержень $(h M): a$ - осевая сила; $\sigma$ - поперечная сила; $в$ - скручивающий момент

Если же рассматривать новый разрабатываемый стержень, то можно отметить следующее. Распределение контактного давления равномерное, что вполне объясняется конструкцией стержня и способом его установки в кости. Величина контактного давления составила порядка 70 МПа и оказалась наибольшей для случая осевого нагружения. Действительно, проксимальный костный отломок давит на нижний, при этом его не удерживают блокирующие винты. В случае поперечной силы величина контактного давления ниже (до 40 МПа). Поперечная сила пытается сместить проксимальный костный отломок в направлении, перпендикулярном оси стержня, что и приводит к уменьшению контактного давления и его менее равномерному распределению по сравнению со случаем осевого нагружения. В третьем случае, когда система кость-фиксатор нагружается скручивающим моментом, контактное давление на 
стыке отломков не больше 2 МПа по величине, но распределено равномерно. Действительно, приложенный к головке кости момент пытается повернуть проксимальный отломок относительно дистального, что дает сравнительно небольшое контактное давление на их стыке. Форма перелома и стержня формирует равномерный контакт двух отломков, за счет чего сохраняется равномерное распределение давления между ними (рис. 6).

Обратимся к перелому $B 2$ и рассмотрим распределение и величину контактного давления между отломками и осколком, возникающего под действием внешних нагрузок (рис. 7-9).
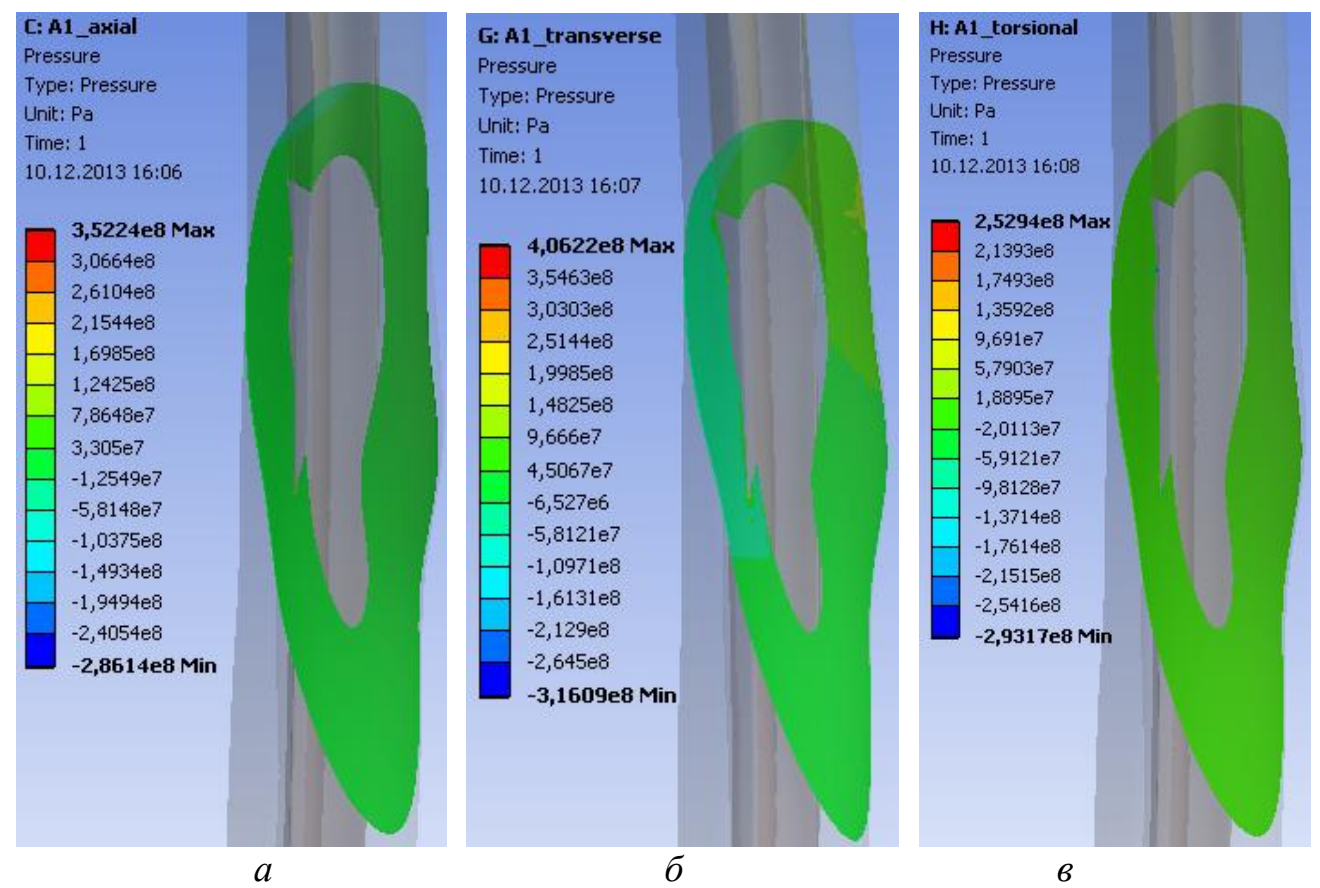

Рис. 6. Контактное давление между отломками кости при приложении осевой $(a)$, поперечной (б), скручивающей (в) нагрузок (перелом $A 1)$
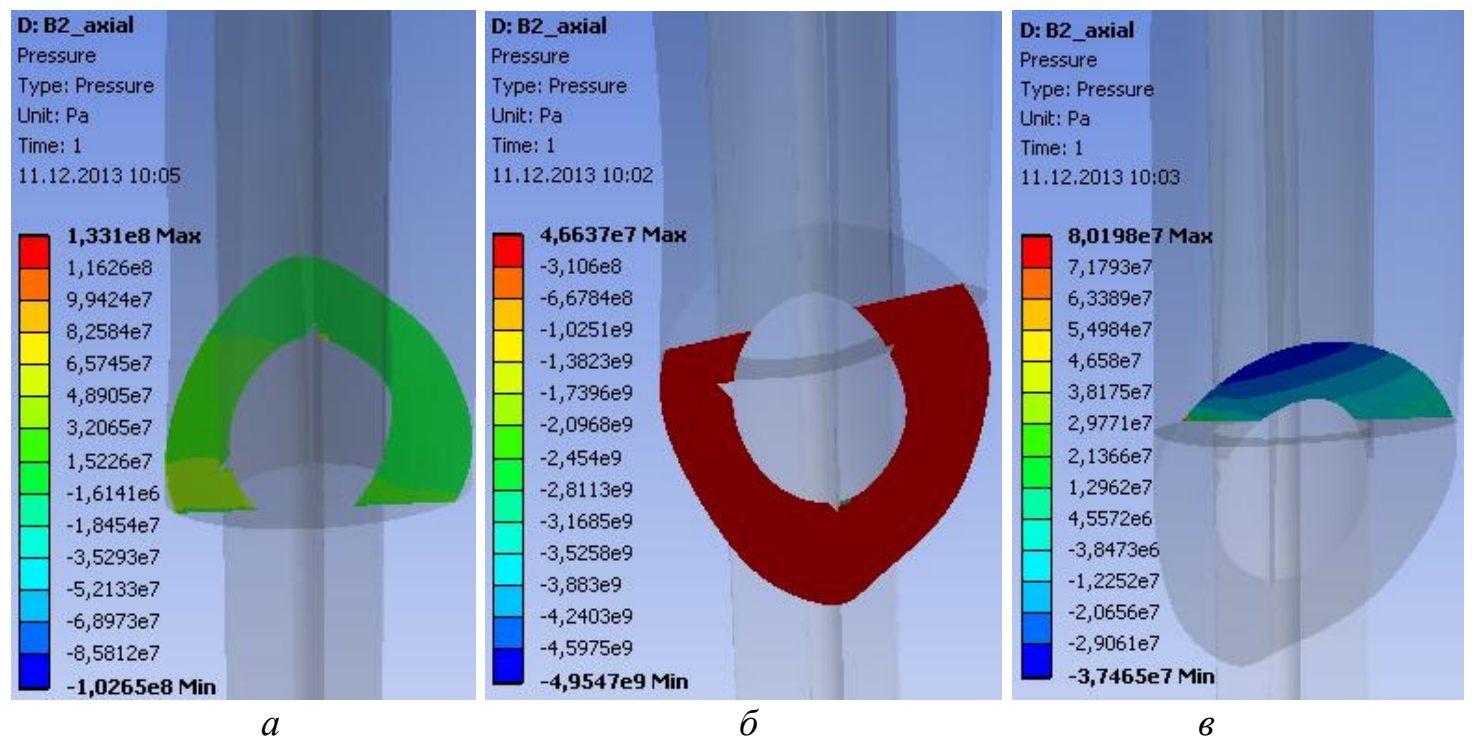

Рис. 7. Контактное давление между отломками кости при приложении осевой нагрузки (перелом $B 2$ ): $a$ - между проксимальным отломком и центральным осколком; $\sigma$ - между центральным осколком и дистальным отломком; $в$ - между

проксимальным и дистальным отломками 


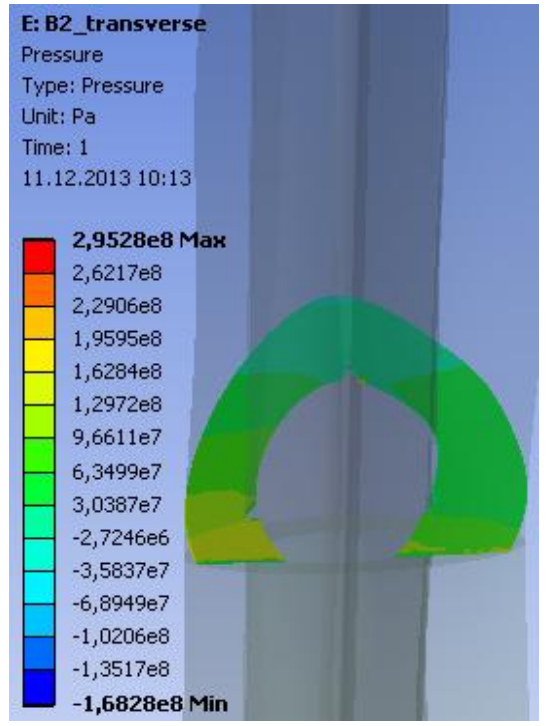

$a$

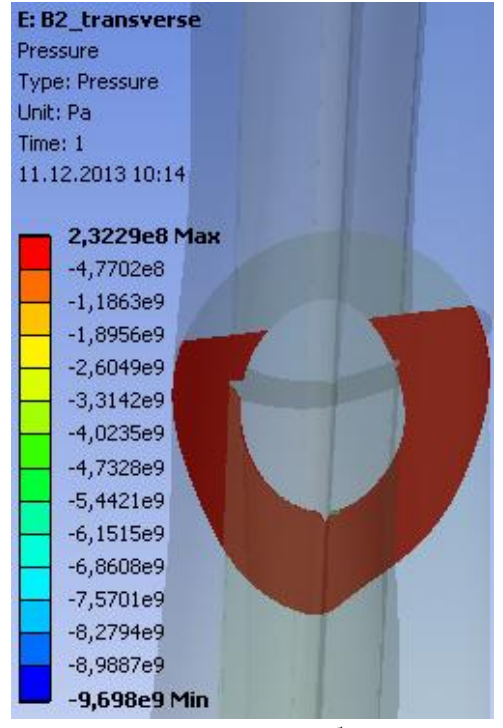

$\sigma$

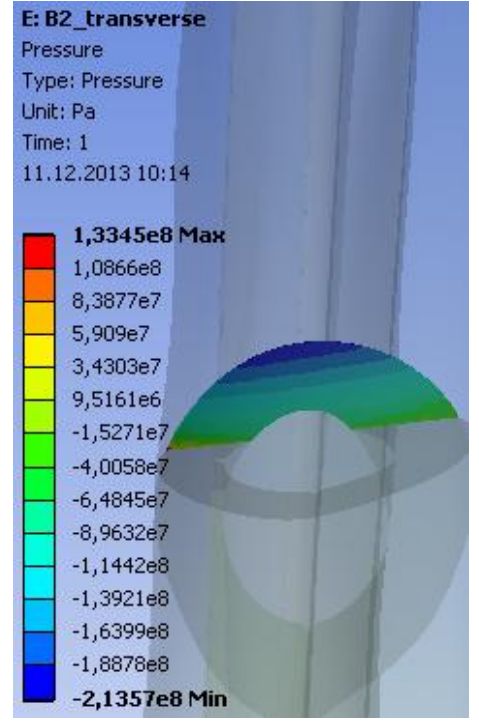

B

Рис. 8. Контактное давление между отломками кости при приложении поперечной нагрузки (перелом $B 2$ ): $a$ - между проксимальным отломком и центральным осколком; $\sigma$ - между центральным осколком и дистальным отломком; в - между проксимальным и дистальным отломками
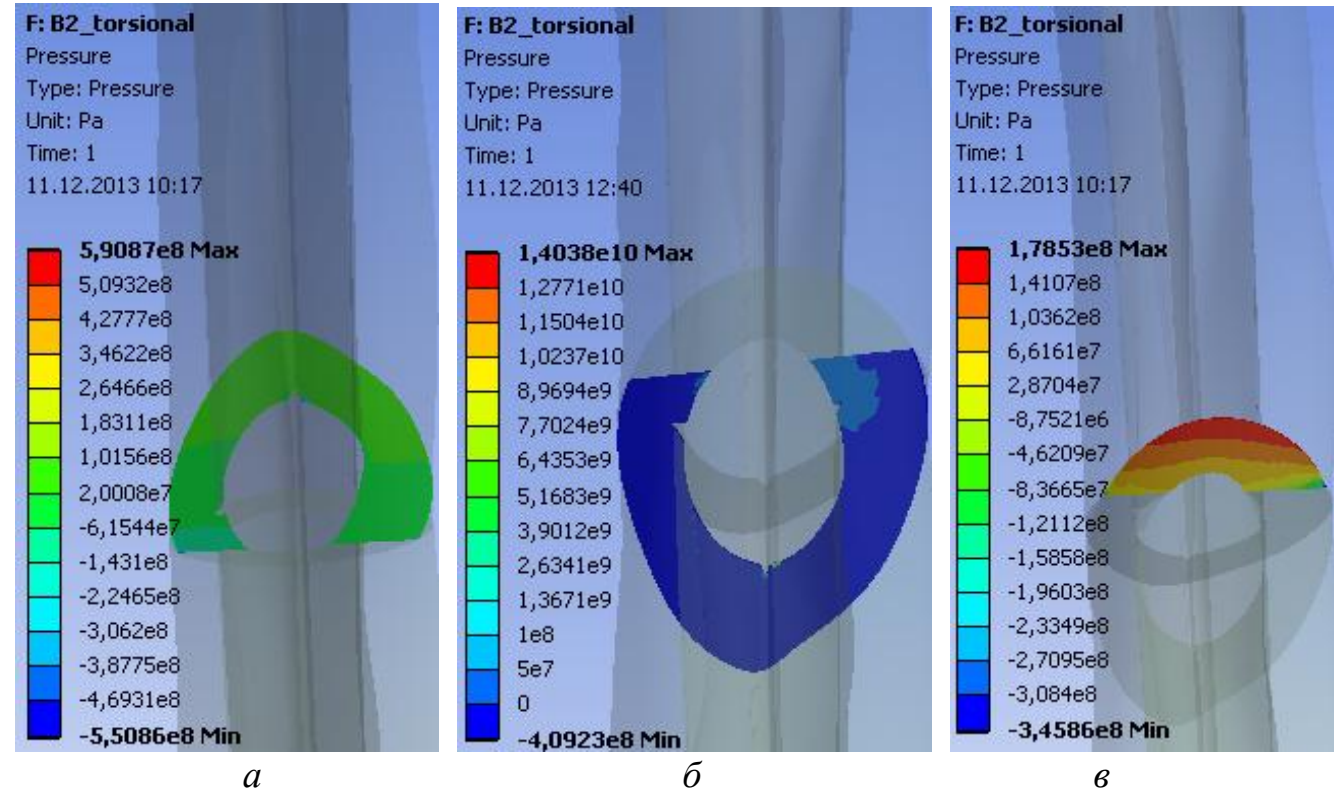

Рис. 9. Контактное давление между отломками кости при приложении скручивающей нагрузки (перелом $B 2$ ): $a$ - между проксимальным отломком и центральным осколком; $\sigma$ - между центральным осколком и дистальным отломком; в - между проксимальным и дистальным отломками

Рис. 7-9, б показывают равномерное распределение контактного давления между центральным осколком и дистальным отломком для всех трех вариантов нагружения. В случае осевого нагружения величина контактного давления не более 45 МПа, в случае поперечного - 230 МПа, в случае скручивающего - 400 МПа. Равномерность распределения контактного давления в этих случаях обеспечивается конструкцией стержня и фиксацией отломков. 
Рис. 7-9, $a$ показывают контактное давление между проксимальным отломком и центральным осколком. Видно, что распределение давления на большей части площади контакта равномерное. В случае осевого нагружения величина контактного давления не более $47 \mathrm{MПа,} \mathrm{в} \mathrm{случае} \mathrm{поперечного} \mathrm{-} 160$ МПа, в случае скручивающего - 100 МПа. Здесь основную роль играет форма проксимального отдела стержня, а также величина поперечного сечения осколка, которые распределяют нагрузку равномерно между отломком и осколком.

На рис. 7-9, в контактное давление между отломками неравномерно. Видно, что на одной части контактной поверхности оно положительно, на другой - отрицательно. Это говорит о разной направленности давления. Клиновидная форма проксимального отломка определяет эту неравномерность и разную направленность. В самом деле, проксимальный отломок давит на линии контакта с осколком за счет своей клиновидной формы и малой площади контакта отломков.

Перейдем к рассмотрению эффективных напряжений, возникающих в системе кость-фиксатор. Сначала рассмотрим стандартный блокируемый стержень $C h M$.

При анализе напряжений, возникающих в стержне ChM, было выявлено следующее. Наибольшие напряжения достигаются как в теле стержня в области перелома, так и на блокирующих винтах, а также на стержне в месте его контакта с блокирующими винтами. Данная картина распределения напряжений характерна для обоих видов переломов и трех исследованных видов нагрузок (рис. 10 и 11).

Величина эффективных напряжений для стандартного стержня достигает 115-120 МПа, что меньше предела упругости нержавеющей стали. Тем не менее картина распределения эффективных напряжений (концентрации на винтах и в месте контакта стержня с винтами) является характерной для данного типа стержней и показывает неудачность его конструкции. Более того, относительно высокие напряжения в месте контакта костных отломков и винтов свидетельствуют о нагруженности этих областей и, следовательно, могут привести к повреждению/разрушению костной ткани.

Перейдем к анализу напряженно-деформированного состояния системы костьимплантат в случае установки стержня нового типа. На рис. 12 изображены поля эффективных напряжений на стержне нового типа в случае осевой нагрузки для переломов $A 1$ и $B 2$. Картины напряжений для других типов нагрузок аналогичны приведенным на рис. 12.
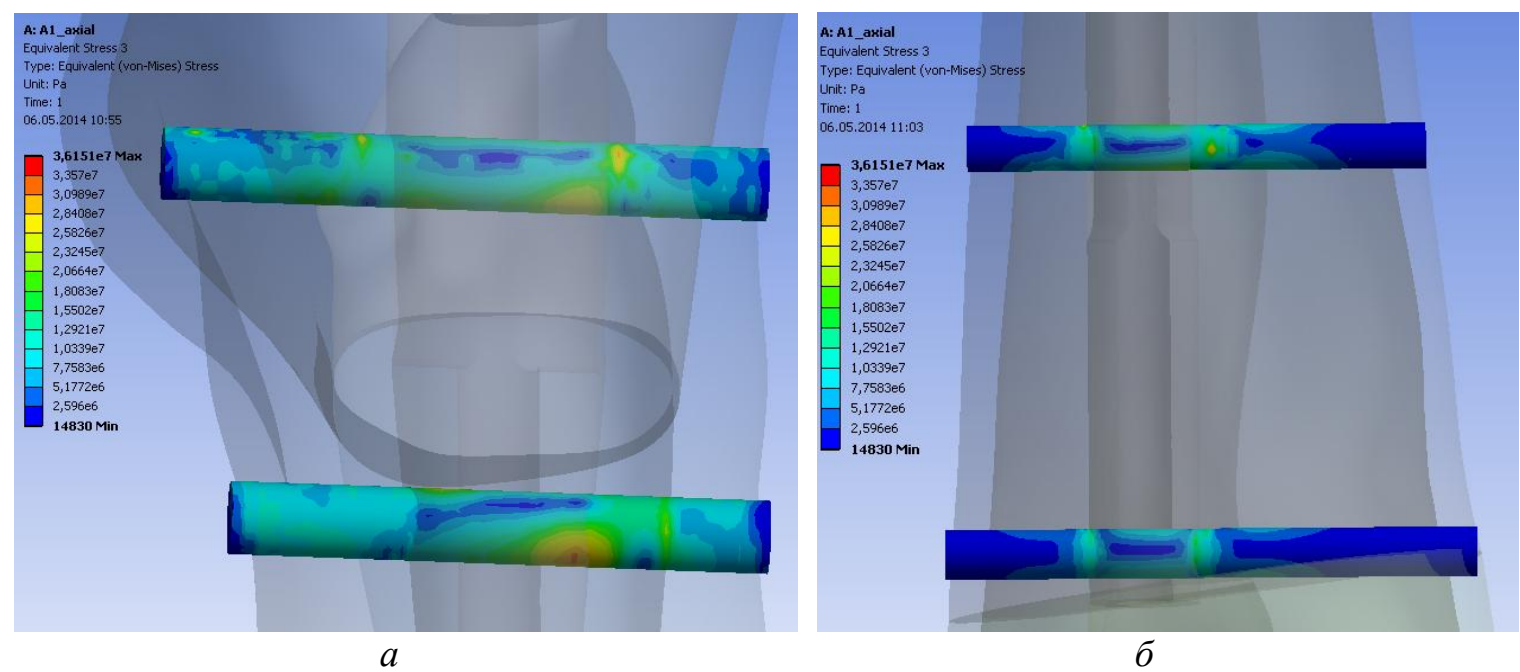

Рис. 10. Эффективные напряжения на проксимальных блокирующих винтах при: $a$ - осевой нагрузке и переломе $A 1 ; \sigma$ - скручивающей нагрузке и переломе $A 1$ 


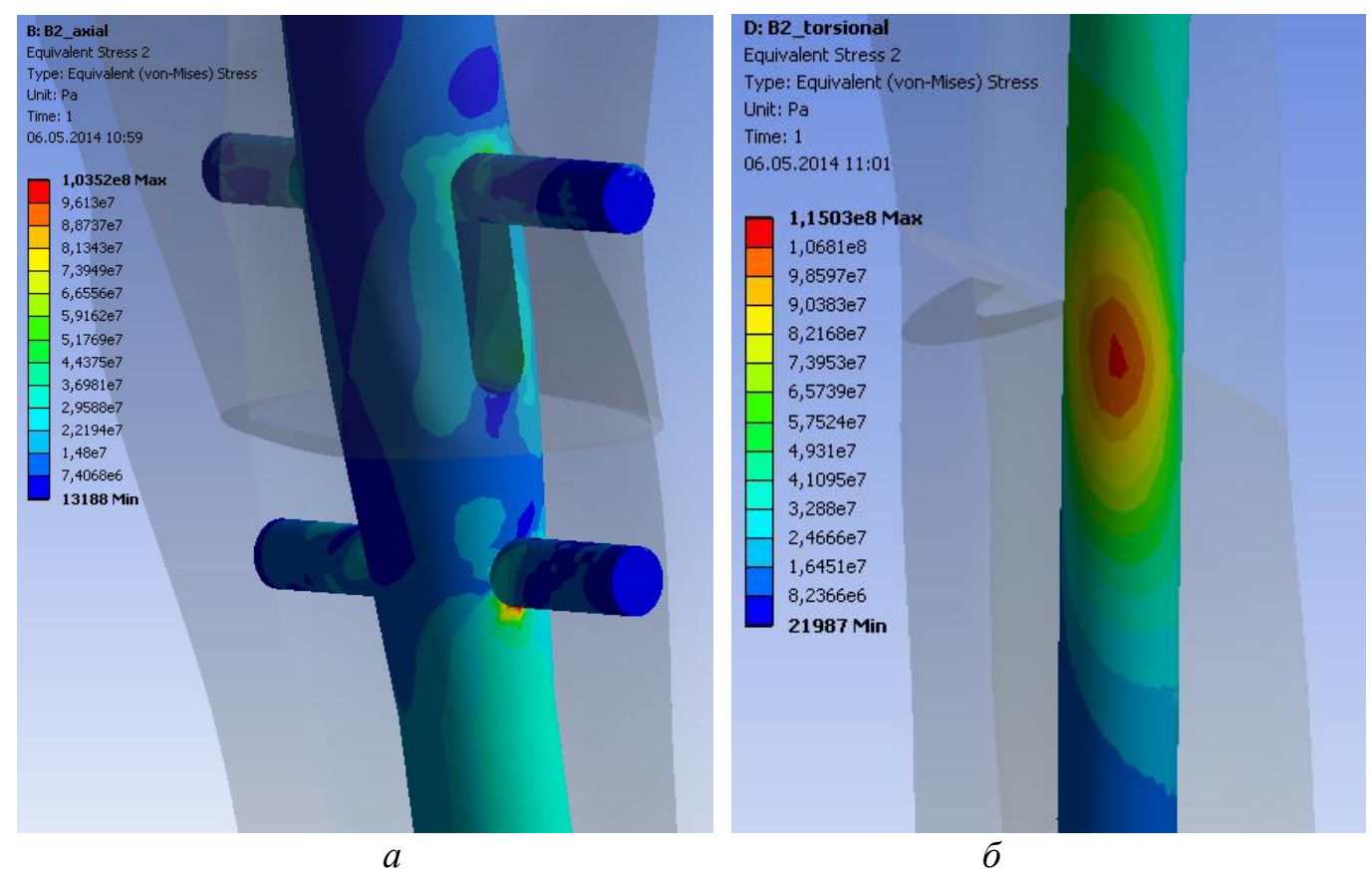

Рис. 11. Эффективные напряжения на проксимальных блокирующих винтах и стержне при: $a$ - осевой нагрузке и переломе $B 2 ; \sigma$ - скручивающей нагрузке и переломе $B 2$

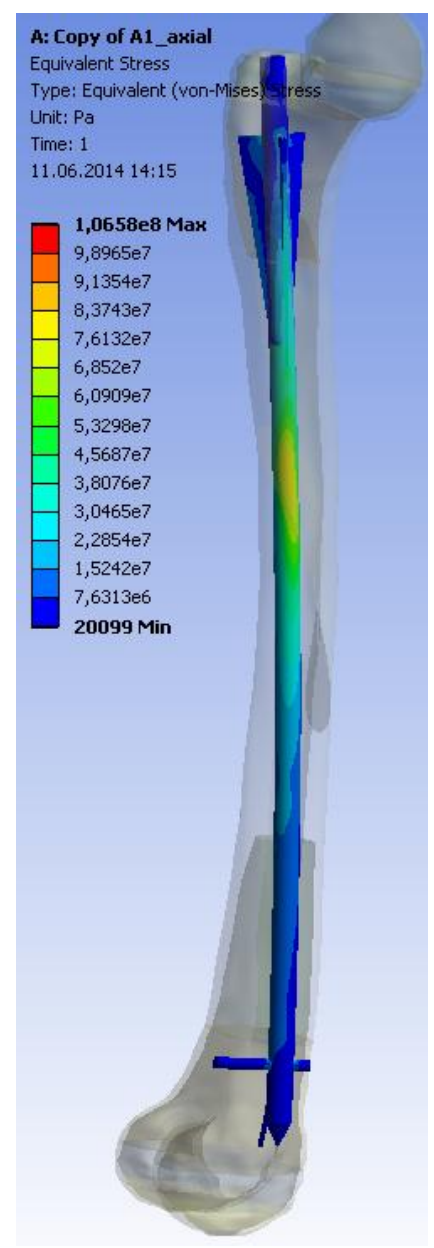

$a$

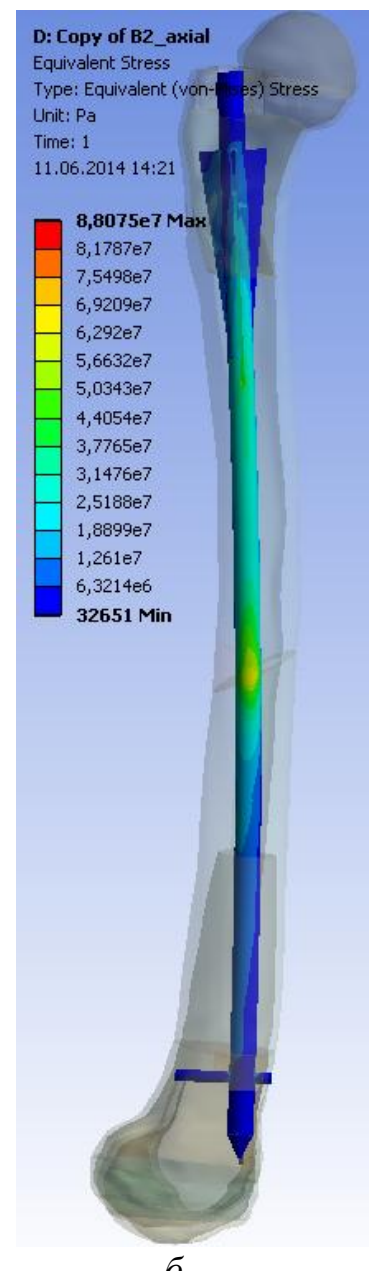

6

Рис. 12. Эффективные напряжения в стержне нового типа в случае осевой нагрузки: $a$ - перелом $A 1 ; \sigma$ - перелом $B 2$ 


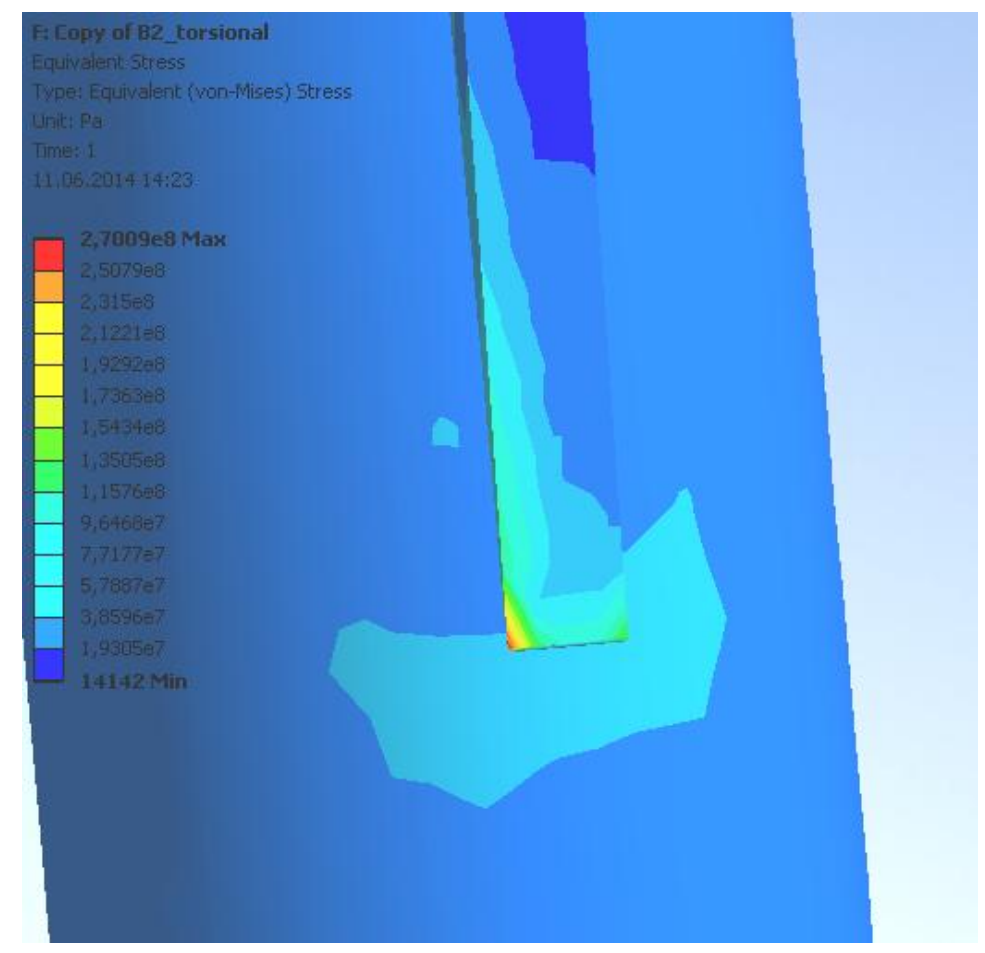

Рис. 13. Локализация эффективных напряжений в случае перелома $B 2$ и поперечной (скручивающей) нагрузки

Если анализировать рис. 12, можно сказать следующее. Напряжения, возникающие в стержне, не превышают значение в 107 МПа. Однако в случае перелома $B 2$ и поперечной, а также скручивающей нагрузки эффективные напряжения в стержне достигают 220-270 МПа и локализуются в нижней точке прикрепления большей лопасти к телу стержня (рис. 13). Величина напряжений в этом случае составляет чуть более модуля упругости нержавеющей стали, но их локализация не представляет опасности для конструкции стержня и объясняется его установкой в данной конкретной кости.

Необходимо отметить, что наибольшие эффективные напряжения возникают на теле стержня, дистальный блокирующий винт и спица существенно менее нагружены. Последний факт свидетельствует о том, что вероятность повреждения и поломки винта существенно снижается. Величина наибольших эффективных напряжений ниже предела текучести нержавеющей стали.

\section{ЗАКЛЮЧЕНИЕ}

Разрабатываемый стержень с точки зрения механики имеет более удачную конструкцию, чем стандартный блокируемый стержень. Это объясняет равномерность контактного давления между отломками, а также более равномерное распределение напряжений в теле стержня. Нижний блокирующий винт оказывается практически свободным от напряжений в случае двух исследованных переломов ( $A 1$ и $B 2$ ) и трех исследованных нагрузок (осевая, поперечная и скручивающая).

Таким образом, применение фиксатора нового типа при остеосинтезе переломов бедренных костей может улучшить прогноз для пациентов, снизит вероятность поломки блокирующих винтов, а также самого стержня. Это позволит избежать послеоперационных осложнений, а также необходимости выполнения повторных операций. Более того, в конструкции нового стержня не предусмотрены проксимальные блокирующие винты, а проксимальный отломок может перемещаться в направлении 
дистального отломка. Последний факт говорит о том, что для стержней такого типа операции по динамизации конструкции (что подразумевает под собой выполнение повторного операционного вмешательства по извлечению одного из проксимальных блокирующих винтов) не будут требоваться.

\section{СПИСОК ЛИТЕРАТУРЫ}

1. Alho A., Benterud J.G., Hogevold H.E., Ekeland A., Stromsoe K. Comparison of functional bracing and locked intramedullary nailing in the treatment of displaced tibial shaft fractures // Clin. Orthop. Relat. Res. - 1992. - Vol. 277. - P. 243-250.

2. AO principles of fracture management. 2nd ed. / ed. by T.P. Ruedi, R.E. Buckley, C.G. Moran. - Stuttgard, New York: Thieme Publishers, 2007. - 1103 p.

3. Blum J., Karagül G., Sternstein W., Rommens P.M. Bending and torsional stiffness in cadaver humeri fixed with a self-locking expandable or interlocking nail system: a mechanical study // J. Orthop. Trauma. 2005. - Vol. 19, № 8. - P. 535-542.

4. Buciu G., Grecu D., Niculescu G., Chiutu L., Stoica M., Popa D. Studies about virtual behavior of tibia fractures and nails during the fixation process // Journal of Industrial Design and Engineering Graphics. 2013. - Vol. 8, № 2. - P. 5-10.

5. Cheung G., Zalzal P., Bhandari M., Spelt J.K., Papini M. Finite element analysis of a femoral retrograde intramedullary nail subject to gait loading // Med. Eng. Phys. - 2004. - Vol. 26, № 2. - P. 93-108.

6. Christie J., Court-Brown C., Kinninmonth A.W., Howie C.R. Intramedullary locking nails in the management of femoral shaft fractures // J. Bone Joint Surg. (Br). - 1988. - Vol. 70, № 2. - P. 206-210.

7. Court-Brown C.M., Christie J., McQueen M.M. Closed intramedullary tibial nailing: its use in closed and type I open fractures // J. Bone Joint Surg. (Br). - 1990. - Vol. 72, № 4. - P. 605-611.

8. Efstathopoulos N., Nikolaou V.S., Xypnitos F.N., Korres D., Lazarettos I., Panousis K., Kasselouris E.N., Venetsanos D.T., Provatidis C.G. Investigation on the distal screw of a trochanteric intramedullary implant (Fi-nail) using a simplified finite element model // Injury. - 2010. - Vol. 41, № 3. - P. 259-265.

9. Helwig P., Faust G., Hindenlang U., Hirschmüller A., Konstantinidis L., Bahrs C., Südkamp N., Schneider R. Finite element analysis of four different implants inserted in different positions to stabilize an idealized trochanteric femoral fracture // Injury. - 2009. - Vol. 40, № 3. - P. 288-295.

10. Hooper G.J., Keddell R.G., Penny I.D. Conservative management or closed nailing for tibial shaft fractures. A randomized prospective trial // J. Bone Joint Surg. (Br). - 1991. - Vol. 73, № 1. - P. 83-85.

11. Ingrassia T., Mancuso A., Ricotta V. Design of a new tibial intramedullary nail // Proc. of the IMProVe 2011 International Conference on Innovative Methods in Product Design. - Venice: Libreria Internazionale Cortina Padova, 2011. - P. 678-684.

12. Kapoor S.K., Kataria H., Boruah T., Patra S.R., Chaudhry A., Kapoor S. Expandable self-locking nail in the management of closed diaphyseal fractures of femur and tibia // Indian J. Orthop. - 2009. - Vol. 43, № 3. - P. 264-270.

13. Lepore L., Lepore S., Maffuli N. Intramedullary nailing of the femur with an inflatable self-locking nail: comparison with locked nailing // J. Orthop. Sci. - 2003. - Vol. 8, № 6. - P. 796-801.

14. Lepore S., Capuano N., Lepore L., Romano G. Preliminary clinical and radiographic results with the Fixion intramedullary nail: an inflatable self-locking system for long bone fractures // J. Orthopaed. Traumatol. 2000. - Vol. 1, № 3. - P. 135-140.

15. Maher S.A., Meyers K., Borens O., Suk M., Grose A., Wright T.M., Helfet D. Biomechanical evaluation of an expandable nail for the fixation of midshaft fractures // J. Trauma. - 2007. - Vol. 63, № 1. - P. $103-107$.

16. Matityahu A., Schmidt A.H., Grantz A., Clawson B., Marmor M., McClellan R.T. The variable angle hip fracture nail relative to the Gamma 3: a finite element analysis illustrating the same stiffness and fatigue characteristics // Advances in Orthopedics. - 2013. DOI: 10.1155/2013/143801.

17. Oliveira M.L., Lemon M.A., Mears S.C., Dinah A.F., Waites M.D., Knight T.A., Belkoff S.M. Biomechanical comparison of expandable and locked intramedullary femoral nails // J. Orthop. Trauma. 2008. - Vol. 22, № 7. - P. 446-450.

18. Ozturk H., Unsaldi T., Oztemur Z., Bulut O., Korkmaz M., Demirel H. Extreme complications of Fixion nail in treatment of long bone fractures // Arch. Orthop. Trauma Surg. - 2008. - Vol. 128, № 3. - P. 301306.

19. Pascarella R., Nasta G., Nicolini M., Bertoldi E., Maresca A., Boriani S. The Fixion nail in the lower limb. Preliminary results // Chir. Organi. Mov. - 2002. - Vol. 87, № 3. - P. 169-174.

20. Rodrigues L.B., Las Casas E.B., Lopes D.S., Folgado J., Fernandes P.R., Pires E.A., Alves G.E., Faleiros R.R. A finite element model to simulate femoral fractures in calves: testing different polymers for intramedullary interlocking nails // Vet. Surg. - 2012. - Vol. 41, № 7. - P. 838-844. 
21. Rose D.M., Smith T.O., Nielsen D., Hing C.B. Expandable intramedullary nails in lower limb trauma: a systematic review of clinical and radiological outcomes // Strat. Traum. Limb Recon. - 2013. - Vol. 8, № 1. - P. 1-12

22. Smith W.R., Ziran B., Agudelo J.F., Morgan S.J., Lahti Z., Vanderheiden T., Williams A. Expandable intramedullary nailing for tibial and femoral fractures: a preliminary analysis of perioperative complications // J. Orthop. Trauma. - 2006. - Vol. 20, № 5. - P. 310-314.

23. Tarnita D., Tarnita D., Bolcu D. Orthopaedic modular implants based on shape memory alloys / ed. Prof. Reza Fazel // Biomedical Engineering - From Theory to Applications. - Rijeka: Sciyo, 2011. - P. 431-468.

24. Turner C.H., Rho J., Takano Y., Tsui T.Y., Pharr G.M. The elastic properties of trabecular and cortical bone tissues are similar: Results from two microscopic measurement techniques // J. Biomech. - 1999. Vol. 32, № 4. - P. 437-441.

25. Zdero R., Bougherara H. Orthopaedic biomechanics: a practical approach to combining mechanical testing and finite element analysis / ed. D. Moratal // Finite Element Analysis. - Rijeka: Sciyo, 2010. P. 171-195.

26. Zhang S., Zhang K., Wang Y., Feng W., Wang B., Yu B. Using three-dimensional computational modeling to compare the geometrical fitness of two kinds of proximal femoral intramedullary nail for chinese femur // Scientific World Journal. - 2013. DOI: 10.1155/2013/978485.

27. Zysset P.K., Guo X.E., Hoffler C.E., Moore K.E., Goldstein S.A. Elastic modulus and hardness of cortical and trabecular bone lamellae measured by nanoindentation in the human femur // J. Biomech. - 1999. Vol. 32, № 10. - P. 1005-1012.

\section{NEW INTRAMEDULLARY NAIL FOR OSTEOSYNTHESIS OF THE FEMUR DIAPHYSEAL FRACTURES}

\section{D.V. Ivanov, A.P. Barabash, Yu.A. Barabash (Saratov, Russia)}

Today, interlocked intramedullary osteosynthesis is the most common treatment of the human lower limb long bones diaphyseal fractures. Modern expandable nails, such as Fixion, have several advantages over standard lockable nails. They are quick to install and provide the necessary compression at the fragments junction. Nevertheless, studies show their low stability in case of torsional loads. Moreover, these nails are substantially more expensive than standard. This paper proposes a new design of intramedullary nail and biomechanical analysis of the femur-nail systems under the influence of three types of loads: axial, lateral and torsional applied to the bone head. Finite element simulation of the stress-strain state of the femur-nail systems was conducted. Two types of bone diaphyseal fractures (oblique and comminuted) and two types of intramedullar nails (new and standard $C h M$ ) were investigated. It was revealed that the developed intramedullary nail provides axial stiffness from 428 to $510 \mathrm{~N} / \mathrm{mm}$ for oblique and comminuted fractures. Results showed a uniform distribution of the contact pressure between the bone fragments and the ability of the proximal fragment to move axially. Distal locking screw at all investigated loads never experienced the critical stress, the main load was distributed on the main body of the nail and its blades. Developed nail has no significant disadvantages of the expanding the locking nails. New nail provides high torsional stability and uniform compression at the fragments junction as well as more intimate contact of bone fragments.

Key words: intramedullary osteosynthesis, finite element modelling, hip fracture, effective stresses, contact pressure. 